\title{
INSTABILITY ANALYSIS OF DEEPWATER RISER WITH FAIRINGS
}

\author{
Mahdi Khorasanchi ${ }^{1}$, Shan Huang \\ Department of Naval Architecture and Marine Engineering \\ University of Strathclyde \\ 100 Montrose Street, Glasgow G4 0LZ, UK
}

\begin{abstract}
The paper investigates the mechanism of instability of deepwater risers fitted with fairings and presents an analytical model to predict the instability onset conditions. The simplified case of a two-dimensional (2D) problem was considered. The governing equations were derived, and the hydrodynamic forces were calculated and the effect of motion in these forces was taken into consideration. The final equations were linearised and an eigenvalue analysis was employed to systematically examine the stability with the emphasis on identifying the critical current speed for a given system. This model was validated against the available test results and showed a good agreement. A parametric study was also carried out. It showed the significant role of the hydrodynamic coefficients as well as mass distribution in the stability of the system.
\end{abstract}

Key words: Riser; Fairing; Instability; VIV

\section{Introduction}

Suppression of the vortex-induced-vibration (VIV) of deepwater risers in ocean currents is an important issue. Various methods have been proposed to control this phenomenon. Among them, outfitting the riser with a VIV suppression device is one of the most prevalent techniques. These devices reduce the VIV in different ways and each has its own advantages and drawbacks. Helical strakes, perhaps the most implemented device, suffer from an increase in the drag force. Riser fairings are capable to mitigate VIV while simultaneously reducing drag by streamlining the fluid current round the riser and consequently weakening the vortices shed aft of the body. They are typically of teardrop geometry, varying in terms of the chord length $c$, thickness $t$, span length and tip and tail details.

Tank tests have revealed that fairings are exposed to severe vibrations when the current velocity exceeds a certain limit. Some designs demonstrated typical VIV response meaning that these sections, though

\footnotetext{
${ }^{1}$ Corresponding author. t: +44 141548 4890, e: mahdi.khorasanchi@strath.ac.uk
} 
streamlined to some extent, were still experiencing vortex shedding while some other designs underwent vibrations with different features from VIV. They exhibited self-induced oscillation or dynamic instability characterised by the increase of responses upon excitation (Ericsson and Reding, 1980; Ikeda et al., 2003; Lee and Allen, 2005; Meyer et al., 1995; Slocum et al., 2004).

Dynamic instability, defined in a classical sense, is the fact that response of a system increases with time which is caused by negative damping in the system (Lee and Allen, 2005). Lee and Allen expound that in the context of VIV, dynamic stability can be described otherwise. As the flow speed increases the VIV motion of a cylinder rises to a certain level, and then the motion interferences with the vortex shedding process and begins to break up the symmetric pattern of alternate vortices. The motion magnitude does not increase even if the flow speed continues to rise, thus the process is self-limiting. When the cylinder is fitted with fairings, they can rotate and form an asymmetric section with respect to flow which entails lift force and may amplify the vibration beyond that of a bare riser. This type of vibration is not self-limiting anymore and the amplitude increases along with the velocity. The frequency of this vibration was reported to be less than the frequency of corresponding vortex shedding (Braaton et al., 2008). In general, as the current speed increases the first peak in the vibrations (Figure 1) is caused by vortex shedding (Blevins, 2001) while the second peak at a higher reduced velocity $U_{r}$ is associated with the instability of a riser fitted with fairings.

Some researchers tried to explain the source of the problem through early separation of boundary layer and stall (Calkins, 1984; Ericsson and Reding, 1980). Accordingly, it was recommended to reduce the angle of fairing contour in the leeside to match the fairing profile to the flow regime (Ericsson and Reding, 1980; Grimminger, 1945). Meyer et al argued that observed instability was due to the fact that the centre of rotation of the fairing was located behind the aerodynamic centre (Meyer et al., 1995). Several methods have been proposed to rectify the problem, e.g. trailing-edge fins or adding vortex generators (Calkins, 1984; Gardner and Cole, 1982; Grant and Patterson, 1977; Meyer et al., 1995). On the other hand, large hydrodynamic damping that some fairings generate (Lee et al., 2004) can be a key reason for their dynamically stable response as well as dominant suppression mechanism (Lee and Allen, 2005).

The instability of fairing has made the design engineers carry out extensive model testing on the stability of each suggested fairing profile, e.g. short fairing or dual fin splitter (Spencer et al., 2007). Therefore, it is vital 
and beneficial to predict the instability onset condition for a given system theoretically in the design phase. An analytical model was proposed to explain the mechanism of instability. This model was based on a simple two-dimensional model of airfoil flutter and did not include the effect of hydrodynamic damping (Slocum et al., 2004). The riser and the fairing were modelled as a single body in this model.

The present study endeavours to develop a more comprehensive analytical model and to take the effect of more parameters into consideration. The classical flutter theories cannot be applied directly because they are based on thin-airfoil theory with essential assumptions to ignore viscosity and thickness (Bertin and Smith, 1998). Moreover, an airplane wing is modelled as a cantilever beam with both flexural and torsional stiffness. On the contrary, riser fairings are designed to freely swing about the riser and therefore, no torsional stiffness exists to restore a distorted fairing to initial condition except the stiffness generated by hydrodynamic forces. Hydrodynamic forces depend on the orientation of fairing relative to flow and therefore in the equation of motion they will be coupled with the terms of the fairing motion. Navier-Stokes equations to define hydrodynamic forces require a numerical solution which masks the analytical feature of the model. Thus, it is necessary to make some simplifying approximations in both spatial and temporal domains.

To remove the effect of three-dimensionality on hydrodynamic forces, 'strip theory' approximation is deployed. Thereby, it is assumed that hydrodynamic characteristics of a 3D fairing are equal to that of a $2 \mathrm{D}$ section and spanwise variations of force are negligible. To eliminate the effect of flow history, the equations of motion will be derived under the assumption of quasi-steady dynamic derivatives. The last step is to assess stability of the system against an infinitesimal disturbance from equilibrium position. It should be noted that in a large proportion of all cases, an adequate definition of flutter properties of a system can be obtained by studying the stability of infinitesimal motions (Bisplinghoff et al., 1996).

The development of the analytical stability model discussed in the following is based on few assumptions outlined below:

- $\quad$ Fairing segments are installed on a vertical top tensioned riser.

- Individual fairing segments are rigid structures and do not experience any deformation.

- Fairing segments are free to rotate about the riser and there is no structural torsion-stiffness. 
- Entrapped water within the fairing shell moves with the fairing as a rigid body.

- 'Strip theory' approximation is employed to reduce the three-dimensionality of hydrodynamic characteristics of fairing to a two-dimensional section.

- ' 'Quasi-steady’ assumption is considered and the effect of flow history is eliminated.

- As observed in the tank tests, motion in-line with the current direction is of very limited amplitude in comparison with cross-flow translation, e.g. $0.6 D$ against $4 D$ where $D$ is the riser diameter (Braaton et al., 2008). Thus, in-line motion has negligible effect on flutter-type instability.

- According to quasi-steady assumption, lift, drag and moment are functions of instantaneous angle of attack (AoA). However, the effects arise from cross-flow translation as well as influences due to time variation of AoA (torsional velocity) are to be considered.

These assumptions impose some limitations on the application of this model. This model will be helpful in determining the threshold velocity at which the instability can occur for a given system of riser and fairing. However, it is not capable of explaining the evolution of unstable motion and its development in subsequent stages. Whether the amplitude of this unstable motion continues to increase or is self-limiting is out of the scope of this model.

The other major limitation of this model is that the hydrodynamic coefficients are assumed to vary only with angle of attack, however, they may be affected by turbulence and vortex shedding too. On the other hand, the quasi-steady assumption requires that the vortex shedding frequency be well above the natural frequency of structure. Although vortex shedding from fairings is not very likely as they are devised to suppress VIV, this condition should be assessed if the fairing still experiences some vortices.

It should be mentioned that this model is based on linearization of hydrodynamic forces. Since real physical phenomenon are not linear, the question always arises how good the linearised theory is as an approximation to the real case, and to what extent of magnitude of the variables concerned the linearised theory is valid. At present, it can only be said that experimental observations show the linearised theory of flutter type instability represents fairly closely the real situation in the neighbourhood of the critical instability speed, provided that the amplitude of motion remains small (Fung, 2002). 


\section{Governing Equations of Motion}

A cross-section of a riser fitted with fairings is shown in Figure 2. The riser is a pipe, possibly covered by buoyancy module, filled with fluid and supported by a spring and damper in cross-flow (CF) direction which depict the contribution of the rest of the riser. As the test reports showed large amplitude vibration in CF direction, it is assumed that the negligible motion in line with the current is unimportant in comparison with CF oscillation (see Section 1). Thus, the riser has only the translational degree of freedom (DOF), $y(t)$. The riser also interacts with the fairing which results in transverse and torsional forces, $F_{\mathrm{y}}^{\text {int }}$ and $F_{\theta}^{\text {int }}$ respectively. Fairing is designed to experience as little torsional friction as possible to easily align itself to current. Therefore, it can be deduced that this friction force can affect the rotational freedom of fairing but induces no tangible deformation in the riser, hence, meaning there is no need to consider another DOF for torsion of the riser.

The fairing is constructed robust enough not to bear any structural deformation induced by hydrodynamic forces (see Section 1). Fairing has two DOFs and while moving transversely with the riser $y(t)$, it rotates independently $\theta(t)$. The interaction of the system with the current is through the fairing and associated hydrodynamic forces including lift, drag, moment and added mass. The fairing may be filled with buoyancy material which is rigid and behaves as a part of the fairing. Nevertheless, there are other types of fairings which are hollow and the sea water penetrates into them. The entrapped water within the fairing's shell is assumed to follow its motion and thus is treated as a rigid body like the previous case (see Section 1).

By constructing the free body diagram of the riser and taking $y(t)$ as the only DOF, the governing equation of motion for riser CF transition is derived,

$$
m_{r} \ddot{y}+C_{y} \dot{y}+k_{y} y=F_{y}^{\text {int }}
$$

where $m_{\mathrm{r}}, k_{\mathrm{y}}$ and $C_{\mathrm{y}}$ are the mass per unit length, stiffness and damping coefficient of the riser respectively.

Two groups of forces act on the fairing, i.e. hydrodynamic forces and reaction forces at interface with the riser which include $-F_{\mathrm{y}}^{\mathrm{int}}$ and $-F_{\theta}^{\mathrm{int}}$. The latter represents the torsional friction of angular oscillation. This friction which absorbs energy and works as a damper is modelled here with a viscous type damping term, 
$-F_{\theta}^{\text {int }}=-C_{\theta} \dot{\theta}$. The free body diagram of fairing leads to two coupled equations for transverse and torsional motions.

$$
\begin{aligned}
& \left(\mathrm{m}_{\mathrm{fr}}+\mathrm{m}_{\mathrm{a}}\right) \ddot{\mathrm{y}}-\left(\mathrm{S}_{\mathrm{x}}+\mathrm{S}_{\mathrm{a}}\right) \ddot{\theta}=\mathrm{F}_{\mathrm{y}}^{\mathrm{H}}-\mathrm{F}_{\mathrm{y}}^{\mathrm{int}} \\
& \left(\mathrm{J}+\mathrm{J}_{\mathrm{a}}\right) \ddot{\theta}-\left(\mathrm{S}_{\mathrm{x}}+\mathrm{S}_{\mathrm{a}}\right) \ddot{\mathrm{y}}=\mathrm{F}_{\theta}^{\mathrm{H}}-\mathrm{C}_{\theta} \dot{\theta}
\end{aligned}
$$

where $m_{\mathrm{fr}}, S_{\mathrm{x}}$ and $J$ are the mass, first mass moment of area and polar mass moment of inertia for fairing respectively. Moreover, $m_{\mathrm{a}}, J_{\mathrm{a}}$ and $S_{\mathrm{a}}$ are respectively the added mass parameters of the fairing for CF transition, angular motion and their mutual effects on each other. The terms $F_{\mathrm{y}}^{\mathrm{H}}$ and $F_{\theta}^{\mathrm{H}}$ show hydrodynamic forces on fairing in $\mathrm{CF}$ and rotational directions.

By combining and re-arranging Eq (1) to Eq (3), the final equations of motion of the whole system are obtained,

$$
\begin{aligned}
& \left(m+m_{a}\right) \ddot{y}+C_{y} \dot{y}+k_{y} y-\left(S_{x}+S_{a}\right) \ddot{\theta}=F_{y}^{H} \\
& -\left(S_{x}+S_{a}\right) \ddot{y}+\left(J+J_{a}\right) \ddot{\theta}+C_{\theta} \dot{\theta}=F_{\theta}^{H}
\end{aligned}
$$

where $=m_{\mathrm{r}}+m_{\mathrm{fr}}$.

Although the direction of current is constant, the fairing oscillates in its cross-section plane and therefore hydrodynamic forces depend on the position of the fairing relative to the current direction, i.e. AoA. More significantly, sea water is a fluid and does not react in a prompt manner to any disturbance caused by the fairing's motion. It means the hydrodynamic forces at a specific time may be influenced by the history of fluid's motion. This adds to the complexity of the issue and makes the analysis face some difficulties. To tackle this issue, it was elaborated in methodology (see Section 1) as to why it is necessary to use the 'QuasiSteady' assumption and remove the flow history. By considering the 'Quasi-steady' assumption, the hydrodynamic forces become a function of instantaneous AoA, $\alpha$. With reference to Figure 3, the instantaneous AoA may be written as, $\alpha=\theta-\beta$, where $\beta=\tan ^{-1}[(\dot{y}-R \dot{\theta}) / U]$. The parameter $R$ is the distance of a reference point from the centre of rotation. It is used to describe the resultant of variations of the AoA over the fairing's circumference which are induced by the angular velocity, $\dot{\theta}$. In fact, angular velocity generates a vertical component of velocity vector which varies over the section by the horizontal 
distance from the pivot point. Naturally, the variation of vertical component of the fairing's velocity induces a variable change in AoA at each point along the section. Thus, the definition of measurable hydrodynamic coefficients at which the whole section stands at a specific AoA is violated here. To adapt this variation of AoA to conventional definition, an approximation simulating the effect of angular velocity on the flow field is required. By considering that the fairing is a symmetric section, a reference point on the chord line at a radius $R$ with respect to the centre of rotation (CR) is chosen. By convention, if $R>0$, the reference point is located aft of the CR.

All hydrodynamic forces on the fairing at an instantaneous distortion angle of $\theta$ must be calculated based on the relative current velocity $U_{\text {rel }}$ and instantaneous incidence angle $\alpha$ (Figure 3). For each DOF, the corresponding force is,

$$
\begin{aligned}
& \mathrm{F}_{\mathrm{y}}^{\mathrm{H}}=\left.\frac{1}{2} \rho \mathrm{U}_{\text {rel }}{ }^{2} \mathrm{C} \mathrm{C}_{\mathrm{L}}\right|_{\alpha} \times \cos \beta-\left.\frac{1}{2} \rho \mathrm{U}_{\text {rel }}{ }^{2} \mathrm{C} \mathrm{C}_{\mathrm{D}}\right|_{\alpha} \times \sin \beta \\
& \mathrm{F}_{\theta}^{\mathrm{H}}=\left.\frac{1}{2} \rho \mathrm{U}_{\text {rel }}{ }^{2} \mathrm{C}^{2} \mathrm{C}_{\mathrm{M}(\mathrm{cr})}\right|_{\alpha}
\end{aligned}
$$

where $C_{\mathrm{D}}, C_{\mathrm{L}}$ and $C_{\mathrm{M}(\mathrm{cr})}$ are drag, lift and moment coefficients respectively, measured at CR with respect to chord length $c$.

Hoerner showed that the lift function of a thick foil with $c / t=0.68$ and 0.70 is almost perfectly linear up to 20 degrees (Hoerner, 1992). He also reported a linear relation between drag and lift coefficients of thick foils (Hoerner, 1965). Thus, for prediction of the instability onset the hydrodynamic coefficients at a small AoA, $\alpha$, can be linearised by the use of Taylor series about the equilibrium position $\alpha=0$. The variable $\alpha$ is a function of velocities and needs to be expanded in Taylor series similarly. It can be easily shown that $\beta \approx[(\dot{y}-R \dot{\theta}) / U]$ and $\alpha=\theta-[(\dot{y}-R \dot{\theta}) / U]$. By substituting $\alpha$ and the linearised hydrodynamic coefficients in Eq. (6) we have,

$$
\begin{aligned}
\frac{1}{2} \rho \mathrm{U}_{\text {rel }}{ }^{2} \mathrm{C}\left(\left.\mathrm{C}_{\mathrm{L}}\right|_{\alpha} \cos \beta-\left.\mathrm{C}_{\mathrm{D}}\right|_{\alpha} \sin \beta\right) & =\frac{1}{2} \rho \mathrm{U}^{2} \mathrm{c}\left(\left.\frac{\partial \mathrm{C}_{\mathrm{L}}}{\partial \alpha}\right|_{\alpha=0}\right) \theta \\
& -\frac{1}{2} \rho \mathrm{Uc}\left(\left.\frac{\partial \mathrm{C}_{\mathrm{L}}}{\partial \alpha}\right|_{\alpha=0}+\left.\mathrm{C}_{\mathrm{D}}\right|_{\alpha=0}\right) \dot{\mathrm{y}} \\
& +\frac{1}{2} \rho \mathrm{RUc}\left(\left.\frac{\partial \mathrm{C}_{\mathrm{L}}}{\partial \alpha}\right|_{\alpha=0}+\left.\mathrm{C}_{\mathrm{D}}\right|_{\alpha=0}\right) \dot{\theta}
\end{aligned}
$$


Similarly, Eq. (7) becomes,

$$
\begin{aligned}
\left.\frac{1}{2} \rho \mathrm{U}_{\mathrm{rel}}{ }^{2} \mathrm{C}^{2} \mathrm{C}_{\mathrm{M}(\mathrm{cr})}\right|_{\alpha}= & \left(\left.\frac{1}{2} \rho \mathrm{U}^{2} \mathrm{c}^{2} \frac{\partial \mathrm{C}_{\mathrm{M}(\mathrm{cr})}}{\partial \alpha}\right|_{\alpha=0}\right) \theta+ \\
& -\left(\left.\frac{1}{2} \rho \mathrm{Uc}^{2} \frac{\partial \mathrm{C}_{\mathrm{M}(\mathrm{cr})}}{\partial \alpha}\right|_{\alpha=0}\right) \dot{\mathrm{y}}+\left(\left.\frac{1}{2} \rho R \mathrm{Rc}^{2} \frac{\partial \mathrm{C}_{\mathrm{M}(\mathrm{cr})}}{\partial \alpha}\right|_{\alpha=0}\right) \dot{\theta}
\end{aligned}
$$

By replacing the hydrodynamic force terms in Eq. (4) and (5) with linearised terms from Eq. (8) and (9) and re-arranging the result, the equations of motion will be in the following form,

$$
\begin{aligned}
& \left(\mathrm{m}+\mathrm{m}_{\mathrm{a}}\right) \ddot{\mathrm{y}}+\left(\mathrm{C}_{\mathrm{y}}+\frac{1}{2} \rho \mathrm{Uc}\left(\left.\frac{\partial \mathrm{C}_{\mathrm{L}}}{\partial \alpha}\right|_{\alpha=0}+\left.\mathrm{C}_{\mathrm{D}}\right|_{\alpha=0}\right)\right) \dot{\mathrm{y}}+\mathrm{k}_{\mathrm{y}} \mathrm{y}-\left(\mathrm{S}_{\mathrm{x}}+\mathrm{S}_{\mathrm{a}}\right) \ddot{\theta}+ \\
& -\frac{1}{2} \rho \mathrm{RUc}\left(\left.\frac{\partial \mathrm{C}_{\mathrm{L}}}{\partial \alpha}\right|_{\alpha=0}+\left.\mathrm{C}_{\mathrm{D}}\right|_{\alpha=0}\right) \dot{\theta}-\left(\left.\frac{1}{2} \rho \mathrm{U}^{2} \mathrm{c} \frac{\partial \mathrm{C}_{\mathrm{L}}}{\partial \alpha}\right|_{\alpha=0}\right) \theta=0 \\
& -\left(\mathrm{S}_{\mathrm{x}}+\mathrm{S}_{\mathrm{a}}\right) \ddot{\mathrm{y}}+\left(\left.\frac{1}{2} \rho \mathrm{Uc}^{2} \frac{\partial \mathrm{C}_{\mathrm{M}(\mathrm{cr})}}{\partial \alpha}\right|_{\alpha=0}\right) \dot{\mathrm{y}}+\left(\mathrm{J}+\mathrm{J}_{\mathrm{a}}\right) \ddot{\theta}+\left(\mathrm{C}_{\theta}-\left.\frac{1}{2} \rho \mathrm{RUc}^{2} \frac{\partial \mathrm{C}_{\mathrm{M}(\mathrm{cr})}}{\partial \alpha}\right|_{\alpha=0}\right) \dot{\theta}+ \\
& -\left(\left.\frac{1}{2} \rho \mathrm{U}^{2} \mathrm{c}^{2} \frac{\partial \mathrm{C}_{\mathrm{M}(\mathrm{cr})}}{\partial \alpha}\right|_{\alpha=0}\right) \theta=0
\end{aligned}
$$

\section{Stability Analysis}

Let $\widehat{Y}=\dot{y}$ and $\widehat{\Theta}=\dot{\theta}$. By substituting them in the equations of motion, we have,

$$
\underbrace{\left[\begin{array}{cccc}
1 & 0 & 0 & 0 \\
0 & \left(\mathrm{~m}+\mathrm{m}_{\mathrm{a}}\right) & 0 & -\left(\mathrm{S}_{\mathrm{x}}+\mathrm{S}_{\mathrm{a}}\right) \\
0 & 0 & 1 & 0 \\
0 & -\left(\mathrm{S}_{\mathrm{x}}+\mathrm{S}_{\mathrm{a}}\right) & 0 & \left(\mathrm{~J}+\mathrm{J}_{\mathrm{a}}\right)
\end{array}\right]}_{\underline{\underline{A}}} \underbrace{\left\{\begin{array}{c}
\dot{\mathrm{y}} \\
\dot{\hat{Y}} \\
\dot{\theta} \\
\dot{\hat{\Theta}}
\end{array}\right\}}_{\underline{\underline{X}}}=
$$

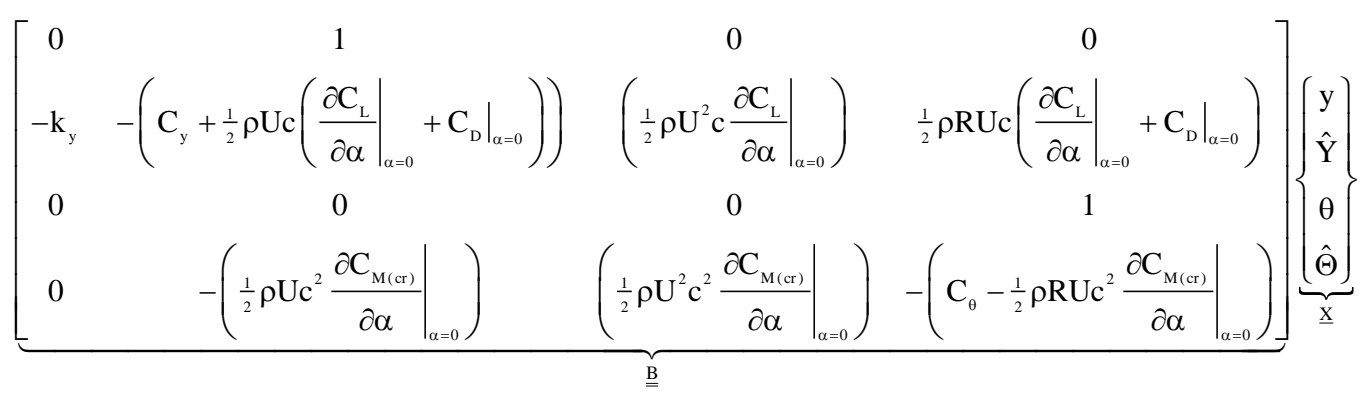

A general solution in exponential form is assumed $\left(\underline{X}=\underline{\mathrm{a}} e^{\lambda \mathrm{t}}\right)$, where $\underline{\mathrm{a}}$ is a vector of constants. By substituting in the above equation, it results in the matrix equation $[\underline{\underline{A}} \lambda-\underline{B}] \cdot \underline{\mathrm{a}}=\underline{0}$. This linear homogenous 
equation has non-trivial solution only if the coefficient matrix is singular which requires the matrix determinant to be zero. More significantly, this solution/response should be stable. The stability of the system dictates that the amplitude of response should decline and the vibration should abate through the time. This means the real part of power in assumed exponential solution should be negative.

By setting the determinant to zero and expanding the terms, it renders a characteristic equation in $\lambda$ in the form of a polynomial of fourth degree, i.e. $\sum_{\mathrm{i}=1}^{4} \mathrm{c}_{\mathrm{i}} \lambda^{\mathrm{i}}=0$. The coefficients $c_{\mathrm{i}}$ are functions of three categories of variables, i.e. structural properties of the riser and fairing, hydrodynamic characteristics of the fairing and finally current velocity. Therefore, for a given system, current velocity is the only variable which governs the instability onset.

Classifying the coefficients of characteristic equation and making them dimensionless assists the designer to have a better understanding of the true physical parameters that influence the stability. Prior to specifying these parameters, it should be noted that in addition to transverse spring stiffness, the last term in the second equation of motion, e.g. Eq. (11), can be interpreted as the hydrodynamic torsional stiffness (for further explanation, see discussion). Thus, angular velocities and natural frequencies of both motions are calculated as below,

$$
\begin{aligned}
& \omega_{\mathrm{y}}=2 \pi \mathrm{f}_{\mathrm{y}}=\sqrt{\frac{\mathrm{k}_{\mathrm{y}}}{\mathrm{m}+\mathrm{m}_{\mathrm{a}}}} \\
& \omega_{\theta}=2 \pi \mathrm{f}_{\theta}=\sqrt{-\left.\frac{1}{2} \rho \mathrm{U}^{2} \mathrm{c}^{2} \frac{\partial \mathrm{C}_{\mathrm{M}(\mathrm{cr})}}{\partial \alpha}\right|_{\alpha=0} /\left(\mathrm{J}+\mathrm{J}_{\mathrm{a}}\right)}
\end{aligned}
$$

In order to make the characteristic equation dimensionless, the following parameters are defined.

$$
\begin{aligned}
& \tilde{\lambda}=\frac{\lambda}{\mathrm{U} / \mathrm{c}} \\
& \gamma^{2}=\frac{\mathrm{J}+\mathrm{J}_{\mathrm{a}}}{\left(\mathrm{m}+\mathrm{m}_{\mathrm{a}}\right) \cdot \mathrm{c}^{2}} \\
& \mathrm{~S}_{\mathrm{r}}=\frac{\mathrm{S}_{\mathrm{x}}+\mathrm{S}_{\mathrm{a}}}{\left(\mathrm{m}+\mathrm{m}_{\mathrm{a}}\right) \cdot \mathrm{c}} \\
& \mathrm{A}=\frac{\rho \cdot \mathrm{c}^{2}}{2\left(\mathrm{~m}+\mathrm{m}_{\mathrm{a}}\right)}
\end{aligned}
$$




$$
\begin{aligned}
& \xi_{\mathrm{y}}=\frac{\mathrm{C}_{\mathrm{y}}}{2 \cdot\left(\mathrm{m}+\mathrm{m}_{\mathrm{a}}\right) \cdot \omega_{\mathrm{y}}} \\
& \xi_{\theta}=\frac{\mathrm{C}_{\theta}}{2 \cdot\left(\mathrm{J}+\mathrm{J}_{\mathrm{a}}\right) \cdot \omega_{\theta}} \\
& \mathrm{U}_{\mathrm{ry}}=\frac{\mathrm{U}}{\omega_{\mathrm{y}} \cdot \mathrm{C}} \\
& \mathrm{U}_{\mathrm{r} \theta}=\frac{\mathrm{U}}{\omega_{\theta} \cdot \mathrm{C}}=\sqrt{\frac{\gamma^{2}}{\left.\mathrm{~A} \frac{-\partial \mathrm{C}_{\mathrm{M}(\mathrm{rr})}}{\partial \alpha}\right|_{\alpha=0}}} \\
& \mathrm{R}_{\mathrm{r}}=\frac{\mathrm{R}}{\mathrm{C}}
\end{aligned}
$$

where the imaginary part of $\tilde{\lambda}$ shows the ratio of the time that takes a flow particle to pass the chord length to the period of oscillation; $\gamma$ is the radius of gyration about pivot point; $S_{\mathrm{r}}$ is the dimensionless distance of centre of gravity from pivot point; $A$ is the inverse of mass ratio; $\xi_{\mathrm{y}}$ and $\xi_{\theta}$ are the damping ratio of transverse and torsional motions in water respectively; $U_{\mathrm{ry}}$ and $U_{\mathrm{r} \theta}$ are the reduced velocities; and $R_{\mathrm{r}}$ is the dimensionless distance of the reference point.

By substituting the above parameters, the dimensionless form of the characteristic equation is as below,

$$
\begin{aligned}
\sum_{\mathrm{i}=1}^{4} \tilde{\mathrm{c}}_{\mathrm{i}} \tilde{\lambda}^{\mathrm{i}}=0 & \\
\tilde{\mathrm{c}}_{4}= & {\left[\gamma^{2}-\mathrm{S}_{\mathrm{r}}^{2}\right] } \\
\tilde{\mathrm{c}}_{3}= & {\left[\left.\mathrm{A}\left(\mathrm{S}_{\mathrm{r}}-\mathrm{R}_{\mathrm{r}}\right) \frac{\partial \mathrm{C}_{\mathrm{M}(\mathrm{cr})}}{\partial \alpha}\right|_{\alpha=0}+\mathrm{A}\left(\gamma^{2}-\mathrm{R}_{\mathrm{r}} \mathrm{S}_{\mathrm{r}}\right)\left(\left.\frac{\partial \mathrm{C}_{\mathrm{L}}}{\partial \alpha}\right|_{\alpha=0}+\left.\mathrm{C}_{\mathrm{D}}\right|_{\alpha=0}\right)+2 \gamma^{2}\left(\frac{\xi_{\mathrm{y}}}{\mathrm{U}_{\mathrm{ry}}}+\frac{\xi_{\theta}}{\mathrm{U}_{\mathrm{r} \theta}}\right)\right] } \\
\tilde{\mathrm{c}}_{2}= & {\left[\frac{\gamma^{2}}{\mathrm{U}_{\mathrm{ry}}{ }^{2}}-\left.\mathrm{A}\left(1+2 \mathrm{R}_{\mathrm{r}} \frac{\xi_{\mathrm{y}}}{\mathrm{U}_{\mathrm{ry}}}\right) \frac{\partial \mathrm{C}_{\mathrm{M}(\mathrm{cr})}}{\partial \alpha}\right|_{\alpha=0}+2 \mathrm{~A} \gamma^{2} \frac{\xi_{\theta}}{\mathrm{U}_{\mathrm{r} \theta}}\left(\left.\frac{\partial \mathrm{C}_{\mathrm{L}}}{\partial \alpha}\right|_{\alpha=0}+\left.\mathrm{C}_{\mathrm{D}}\right|_{\alpha=0}\right)+4 \gamma^{2} \frac{\xi_{\mathrm{y}}}{\mathrm{U}_{\mathrm{ry}}} \frac{\xi_{\theta}}{\mathrm{U}_{\mathrm{r} \theta}}+\right.} \\
\tilde{\mathrm{c}}_{1}= & {\left.\left.\left[-\left.\mathrm{AS} \frac{\partial \mathrm{C}_{\mathrm{r}}}{\partial \alpha}\right|_{\alpha=0}\right] 2 \frac{\xi_{\mathrm{y}}}{\mathrm{U}_{\mathrm{ry}}}+\frac{\mathrm{R}_{\mathrm{r}}}{\mathrm{U}_{\mathrm{ry}}{ }^{2}}\right)\left.\frac{\partial \mathrm{C}_{\mathrm{M}(\mathrm{cr})}}{\partial \alpha}\right|_{\alpha=0}+2 \frac{\gamma^{2}}{\mathrm{U}_{\mathrm{ry}}{ }^{2}} \frac{\xi_{\theta}}{\mathrm{U}_{\mathrm{r} \theta}}-\left.\left.\mathrm{A}^{2} \frac{\partial \mathrm{C}_{\mathrm{M}(\mathrm{cr})}}{\partial \alpha}\right|_{\alpha=0} \mathrm{C}_{\mathrm{D}}\right|_{\alpha=0}\right] } \\
\tilde{\mathrm{c}}_{0}= & {\left[-\left.\mathrm{A} \frac{1}{\mathrm{U}_{\mathrm{ry}}{ }^{2}} \frac{\partial \mathrm{C}_{\mathrm{M}(\mathrm{cr})}}{\partial \alpha}\right|_{\alpha=0}\right] }
\end{aligned}
$$

The characteristic Eq. (24) is solved numerically for a given system to find the current velocity at which the system becomes unstable, i.e. the critical condition with $\operatorname{Real}(\tilde{\lambda})=0$. To this end, Eq. (24) is solved for a 
small velocity and the stability of roots is checked by assessing the sign of their real part. If all roots are stable, then the equation is solved again for an increment in velocity and roots are investigated accordingly. This loop is continued and the trend of roots variation against velocity increase is tracked down until either the real part of one of the roots becomes positive and system goes unstable or the magnitude of current velocity exceeds the possible limit in reality.

\section{Verification}

A series of cylinder tests was carried out by ExxonMobil at David Taylor Model Basin (Slocum et al., 2004). One of the tests was on a rigid cylinder with the fairing shown in Figure 4.

In part of these tests, a submerged horizontal spring mounted cylinder was towed through still water while transverse motion was measured. A rigid test cylinder with a diameter of $22 \mathrm{~cm}$ and a length of $390 \mathrm{~cm}$ was fitted with six independent fairing segments, each with a span of $61.2 \mathrm{~cm}$, a chord length of $52.6 \mathrm{~cm}$, and a maximum thickness of $23.2 \mathrm{~cm}$. This system can be modelled with a fairing and a transverse spring as explained earlier. Based on the measured specifications of the test, the dimensionless parameters are evaluated as $A=0.8435, \gamma^{2}=0.0792$ and $S_{\mathrm{r}}=0.2016 . R_{\mathrm{r}}$ is a reference length for average effect of angular velocity on the angle of attack. For a thin airfoil which rotates about its elastic axis, $R_{\mathrm{r}}$ is chosen to give the AoA at a point three-quarters of the way back from the leading edge (Fung, 2002). For a faring, $R_{\mathrm{r}}$ is estimated to be in the range of $\left[0, \frac{3}{4}-\frac{1}{2} t / c\right]$ (Khorasanchi, 2009). The ratio of $c / t$ is equal to 2.267 in this example and therefore, $R_{r}$ is at most 0.53 for a flat plate. In an airfoil it reduces and is selected as $R_{\mathrm{r}}=0.40$. With respect to damping, some experimental tests report high level of in-water damping ratio for riser fitted with fairing, e.g. 0.10 to 0.18 (Lee et al., 2004). However, as the riser is rigid in this test and does not experience any tangible deformation, its contribution to the transverse damping of the system is smaller. Thus, half of the reported value in the technical literature was deployed in calculation, i.e. $\xi_{\mathrm{y}}=0.05$. Moreover, as the tests emphasise on the rotational freedom of fairing, the positive role of torsional friction damping is ignored in favour of being on the safe and conservative margin, i.e. $\xi_{\theta}=0.0$. The last required data is the hydrodynamic coefficients of the fairing. The lift-curve slope and mean drag at the aerodynamic centre were selected according to the reported data. These coefficients need to be scaled to the chord length

of the fairing based on Eq. (6) and (7), i.e. $\left.\frac{\partial C_{L}}{\partial \alpha}\right|_{\alpha=0}=1.146\left(\frac{1}{\text { radian }}\right)$ and $\left.C_{D}\right|_{\alpha=0}=0.176$. Lift-curve slope, 
measured at the aerodynamic centre, was shifted to the centre of rotation (CR) to compute the moment-curve slope, i.e. $\left.\frac{\partial \mathrm{C}_{\mathrm{M}(\mathrm{cr})}}{\partial \alpha}\right|_{\alpha=0}=-0.0344\left(\frac{1}{\text { radian }}\right)$.

The critical reduced velocity, $U_{\mathrm{cr}}$, at which the real part of a solution to the characteristic Eq. (24) becomes positive, is obtained numerically by increasing the reduced velocity and solving this equation at each step. For this case study, the analytical model shows the system becomes unstable at $U_{\text {cr }}=U_{\text {ry(critical) }}=0.51$, which is located in the range of test results (0.42-0.56) (Slocum et al., 2004). This demonstrates the good agreement of the theoretical model with previous experiment.

The trace of eigenvalues is illustrated in Figure 5. The red circles in this figure show the eigenvalues corresponding to the highest velocity at which a pair of them crosses the imaginary axis and their real part becomes positive. According to Eq. (15) and (21), the imaginary and real parts of roots are multiplied by $U_{\text {ry }}$ to remove the effect of non-dimensionalisation. Hence, the horizontal and vertical axes in this figure are $\operatorname{Real}\left(\lambda / \omega_{\mathrm{y}}\right)$ and $\operatorname{Imag}\left(\lambda / \omega_{\mathrm{y}}\right)$ respectively. The imaginary part of the eigenvalue, $\operatorname{Imag}(\lambda)$, represents the frequency of vibration while the real part shows the trend of amplitude variation.

As the governing equations are coupled, the relevant eigenvector or mode shape of each eigenvalue is coupled in the sense it has elements in both DOFs. Thus, it is not possible to attribute the modes into pure transverse or torsion motion.

\section{Parametric Study}

To investigate the effect of key parameters on the critical velocity, a typical drilling riser was selected. Grant and Patterson (1977) performed a series of wind-tunnel tests on two fairing sections for this riser. The chord length $c$ to thickness $t$ ratio of the selected fairing was fixed at 2. This fairing with the total thickness of $1.016 \mathrm{~m}$ embraced the riser of $0.609 \mathrm{~m}$ diameter plus the choke and kill lines. No buoyancy module was used and sea water filled the gap between the riser and fairing's shell. The fairing was fabricated from $0.0034 \mathrm{~m}$ (10 gauge) steel. The riser carries drilling mud with the density of $1795.97 \mathrm{~kg} / \mathrm{m}^{3}$. The effects of the choke and kill lines are ignored in this example. Since nothing was mentioned in the paper about the wall thickness of steel riser, it is assumed to be in the typical range of $0.04 \mathrm{~m}$. 
With reference to the definition of dimensionless parameters and considering the typical geometry of the selected fairing with blunt end and fins, the structural properties are calculated easily as $\gamma^{2}=0.0853, S_{\mathrm{r}}=$ 0.1908 and $A=0.3992$. No data was reported in Grant and Patterson's research about the damping properties of the system. Hence, with reference to a study on short fairings (Lee et al., 2004), damping coefficients are selected in the lower bound, i.e. $\xi_{\mathrm{y}}=0.05$ and $\xi_{\theta}=0.01$. This results in a lower critical current velocity. The fairing was tested in the wind-tunnel (Grant and Patterson, 1977) and the required hydrodynamic coefficients can be extracted from the reported curves as $\left.C_{\mathrm{D}}\right|_{\alpha=0}=0.09,\left.\frac{\partial \mathrm{C}_{\mathrm{L}}}{\partial \alpha}\right|_{\alpha=0}=3.05\left(\frac{1}{\text { radian }}\right)$ and $\left.\frac{\partial \mathrm{C}_{\mathrm{M}(\mathrm{cr})}}{\partial \alpha}\right|_{\alpha=0}=-0.24\left(\frac{1}{\text { radian }}\right)$. The last hydrodynamic property to obtain is the reference length of $R_{r}$. The ratio of $c / t$ is equal to 2.0 and $R_{r}$ is at most 0.50 for a flat plate. It reduces in a fairing and thus is selected here again as 0.40 .

The analytical model predicts the system goes unstable at $U_{c r}=U_{r y}=0.43$. In the following, the set of parameters corresponding to the above example is selected and a parametric study is carried out to identify how the variation of these parameters influences the threshold of instability. Each parameter varies in the range of half to five times of the present value unless otherwise is stated. The red circle in following figures shows the base case.

Figure 6 shows that the instability onset is not particularly sensitive to the drag coefficient. The drag coefficient came into play through the process of finding instantaneous angle of attack (AoA) and emerged in hydrodynamic damping terms. The undesirable effect of drag on $U_{c r}$ is negligible perhaps because it is the sum of drag and lift slope which forms a term in hydrodynamic damping. Moreover, the value of drag in this example is much less than the lift slope and therefore its impact is not tangible. Lift force as observed in Figure 7 has an adverse effect on stability of the system. In fact, the threshold of stability reduces as the slope of the lift coefficient increases.

The moment coefficient displays both a positive and negative role in instability. In Figure 8, as the absolute value of $\partial C_{M} / \partial \alpha$ rises, the critical reduced velocity can increase or decline based on where the initial value is. It will be discussed later that $\partial C_{M} / \partial \alpha<0$ is a necessary condition for stability and when it is violated, the system is unstable. Further investigation confirms this issue in this example and shows that the real part of one of the solutions is positive for a very small current speed if $\partial C_{M} / \partial \alpha>0$. In addition, it was observed that 
if the magnitude (absolute value) $\partial C_{M} / \partial \alpha$ becomes large enough, it makes the system stable for a normal range of $U_{\mathrm{cr}}$. In this example, for large magnitude the system was still stable while $U_{\mathrm{cr}}$ increased up to 6 .

It was mentioned earlier that the parameter $\mathrm{R}_{\mathrm{r}}$ will be in the range of $0<R_{r}<3 / 4-1 / 2 t / c$. In this example with the length to chord ratio of 2, $R_{\mathrm{r}}$ cannot exceed 0.5. Therefore, in the parametric study shown in Figure 9, $R_{\mathrm{r}}$ varies between 0.5 to 1.5 times the base value $\left(R_{\mathrm{r}}=0.40\right)$. The upper limit is selected beyond physical limit merely to investigate the effect of this parameter more clearly. Figure 9 indicates that the critical reduced velocity increases and then decreases as the length of $R_{\mathrm{r}}$ extends.

Parameter $A$ is equal to the inverse of mass ratio of a square section with a side as long as the chord. Therefore, it is proportional to the ratio of fluid mass to structural mass. It is also known that, in general, as the ratio of fluid mass to structural mass increases so does the inclination toward flow-induced vibration. In this example, Figure 10 is in conformity with this principle and confirms the above point. It demonstrates that as $A$ rises, the critical reduced velocity declines.

According to Figure 11, the parameter $\gamma$ which is a dimensionless symbol of the radius of gyration about the pivot axis has a positive influence on $U_{\mathrm{cr}}$. This shows that as the distribution of mass increases its distance from the pivot point, the system becomes unstable at a higher velocity. One simple conclusion may be that moving the buoyancy material to a farther distance or as reported in the literature, adding mass to the tail of the fairing, like bumps and fins, improves the stability.

With reference to the definition of $S_{\mathrm{r}}$, the fraction of $\left(S_{\mathrm{x}}+S_{\mathrm{a}}\right) /\left(m+m_{\mathrm{a}}\right)$ implies the longitudinal distance of the gravity centre of all rotating masses including added mass effect from pivot axis. Hence, $S_{\mathrm{r}}$ is a dimensionless description of this distance with respect to chord length. On the other hand, the parameter $S_{\mathrm{r}}$ shows how two DOFs are coupled inertially (see Discussion). Figure 12 says that as the level of coupling decreases and the two DOFs become inertially independent, critical reduced velocity is moved to higher values and the stability is strengthened.

In other words, this instability is made from a combination of the torsional and transverse modes with phase and amplitude that gain energy from the flow and when either mode acts alone, the system is stable. Blevins (2001) explained this through the natural frequency. He showed that at the onset of instability, there is a tendency for natural frequencies of both transverse and torsional modes to unite to form a single frequency- 
coupled mode that does not exist without flow. Based on the present study, one way to hinder the instability of fairing is to reduce $S_{\mathrm{r}}$ and coupling by moving the mass centre towards the pivot point. This idea is called "mass balancing” in aerodynamics. In addition, this study showed that beyond a certain amount of $S_{\mathrm{r}}$, for instance 0.292 in this example, the system is unstable for any small current velocity. Thus, in Figure $12 S_{\mathrm{r}}$ varies within $10 \%$ to $150 \%$ of the base value.

As expected, structural damping in both forms, transvers and torsion, improves the stability span and delays the instability to a higher current velocity (Figure 13 and Figure 14 ). In this figure damping ratio varies between 0.5 to 10 times of the base value as this value was very small.

\section{Discussion}

It should be noted that the governing Eq. (10) and (11) are coupled in two ways. These equations are coupled inertially through the term $\left(S_{\mathrm{x}}+S_{\mathrm{a}}\right)$, the first mass moment of area. This demonstrates how the acceleration in one DOF affects the inertia of the other DOF. They are also interrelated hydro-dynamically through the angle of attack. It is due to the fact that hydrodynamic coefficients, which exist in both equations, were written in a linear form of AoA. In addition, AoA is influenced by the distortion angle as well as both transverse and torsion velocities, $\dot{y}$ and $\dot{\theta}$. Thus, these terms as a part of AoA emerge in these equations and inter-relate them thoroughly.

Through the process of expanding hydrodynamic coefficients at a small AoA, a number of hydrodynamic terms contributing to stiffness and damping appeared in the governing Eq. (10) and (11). These terms expounds how the hydrodynamic characteristics of a fairing can change the behaviour of the system.

For example, it is obvious that if the system absorbs energy instead of dissipating that through damping, the amplitude of vibration rises continuously and systems goes unstable. In other words, if one of the damping coefficients becomes negative, it means part of the system is gaining energy and, depending on its extent, it can provide the ground for potential instability. Therefore, negative damping can be interpreted as an alert

for the risk of instability. Back to these equations, the two terms of $\frac{1}{2} \rho R U c\left(\left.\frac{\partial \mathrm{C}_{\mathrm{L}}}{\partial \alpha}\right|_{\alpha=0}+\left.C_{\mathrm{D}}\right|_{\alpha=0}\right) \dot{\theta}$ and $\left(-\left.\frac{1}{2} \rho U c^{2} \frac{\partial \mathrm{C}_{\mathrm{M}(\mathrm{cr})}}{\partial \alpha}\right|_{\alpha=0}\right) \dot{y}$ in Eq. (10) and (11) respectively are parts of the hydrodynamic contribution of the fairing to damping. Consequently, one can say that having a positive coefficient in these terms is a necessary 
condition for stability of the system, i.e. $0<\left(\left.\frac{\partial \mathrm{C}_{\mathrm{L}}}{\partial \alpha}\right|_{\alpha=0}+\mathrm{C}_{\mathrm{D}} \mathrm{l}_{\alpha=0}\right)$ and $0<-\left(\partial \mathrm{C}_{\mathrm{M}(\mathrm{cr})} /\left.\partial \alpha\right|_{\alpha=0}\right)$, whereas having no roots with a positive real part for the characteristic Eq. (24) is the sufficient condition, i.e. $U_{\text {ry }}<$ $U_{\mathrm{cr}}$.

The third point relates to yet another contribution of the hydrodynamic coefficients and it should be noted that the coefficient of the last term in Eq. (11), $-\left(\left.\frac{1}{2} \rho U^{2} c^{2} \frac{\partial \mathrm{C}_{\mathrm{M}(\mathrm{cr})}}{\partial \alpha}\right|_{\alpha=0}\right) \theta$, can be interpreted as the only torsional stiffness which is generated by moment coefficient of the fairing. Thus, if this stiffness is positive, which in fact is identical to satisfying one of the necessary conditions above, the moment generated by fluid force on the fairing, twists it back and helps the fairing with self-alignment to the current. However, in case of negative torsional stiffness, i.e. $-\left(\partial \mathrm{C}_{\mathrm{M}(\mathrm{cr})} /\left.\partial \alpha\right|_{\alpha=0}\right)<0$, any infinitesimal rotation from the rest will develop further. In this case, the zero AoA will not be the equilibrium position any more. The equilibrium position as shown in Figure 15 will be at an angle $\alpha_{2}$ at which the moment coefficient is zero and the slope of moment curve is negative.

Since the fairing is a symmetric section, therefore its lift and moment coefficients are counter-symmetric. Thus, instead of one single point of equilibrium at zero AoA, there exist two identical equilibrium positions at AoA of $\alpha_{2}$ and $-\alpha_{2}$. In this case, at any infinitesimal twist, say $0^{+}$, the slope of moment curve is positive and consequently the rotation develops in the positive direction of AoA up to angle $\alpha_{1}$. At this point, the moment is still positive and hence twists the fairing in the positive direction further while the magnitude of moment reduces. This continues up to the point $\alpha_{2}$. At this point, there is no moment to rotate the fairing and, moreover, the slope of the curve is negative and any disturbance will be restored by generation of appropriate counter-moment. It means $\alpha_{2}$ and likewise its counterpart $-\alpha_{2}$ are the equilibrium positions. In the case of any disturbance, the fairing may switch between these two equilibrium positions depending on the strength of counter-moment. This shift and transition between equilibrium positions resemble the fishtailing as several studies reported that. Thus, if any modification to the fairing's section, e.g. adding fins, can resolve the issue of moment coefficient, then the problem of misalignment, and one of the likely causes for fishtailing, will be sorted out. Therefore, it can be concluded that these issues are separate and independent of instability and can occur while the fairing is statically stable at its equilibrium positions rather than zero AoA. In other words, misalignment and perhaps fishtailing in one hand and instability in the other 
hand are two different mechanisms with different governing parameter; one by the moment coefficient and the other by the characteristic equation.

Identifying the hydrodynamic stiffness has another interesting outcome. More scrutiny of the last term in Eq. (11) reveals that torsional stiffness is proportional to the square of current velocity. It means that as a result of any rise in velocity, the system becomes stiffer torsionally and it is therefore expected that the frequency of torsional vibration increases too. On the other hand, it should be noted that the imaginary part of the eigenvalue, $\operatorname{Imag}(\lambda)$, represented the frequency of vibration. This feature is illustrated in Figure 5 where the imaginary part of two conjugate eigenvalues on the left branches is shown to be increasing. In fact, as the current speed increases, the associated frequency of these eigenvalues rises too. Moreover, as the current speed starts from nearly zero in Figure 5, the only torsional stiffness which is due to hydrodynamic force and proportional to the square of current velocity is therefore very small and almost zero. Thus, since there is no torsional stiffness, the frequency of vibration should be zero as well. Figure 5 confirms that the left branches commence from the origin point where the frequency and amplitude are zero.

\section{Conclusion}

In this study, a two-dimensional problem of a fairing on a rigid riser was considered. A two-degree-offreedom model was developed, i.e. cross-flow translation of the riser and fairing as well as the angular rotation of the fairing. Hydrodynamic forces are dependent on instantaneous AoA and therefore, the effect of motion, both transverse and torsional, on AoA was considered. To assess the instability onset conditions, an infinitesimal disturbance from the equilibrium position was assumed to track the tendency of vibration amplitude. Within this small interval, the variation of hydrodynamic coefficients was reasonably postulated to be linear. Finally, an eigenvalue analysis was carried out to clarify when the system goes unstable. The characteristic equation was made dimensionless to present the significance of physical parameters. It was also highlighted that the governing equations are coupled in two ways, inertially and hydro-dynamically. Moreover, the effect of hydrodynamic damping emerged as a necessary condition for stability. In light of the hydrodynamic stiffness, it was explained that misalignment and perhaps fishtailing in one hand and instability in the other hand are different mechanisms with different governing parameters and should not be confused. 
A parametric study was also carried out. The hydrodynamic coefficients showed a significant role in the stability of the system. The lift coefficient had an adverse effect while the moment coefficient and parameter $R_{\mathrm{r}}$, depending on their value, could have positive or negative influences. The parametric study confirmed this principle that the increase of the mass ratio improves the stability. Moreover, growth in radius of gyration resulted in a higher critical velocity. This showed the benefit of mass distribution in stability. It was also demonstrated that as two DOFs become more independent inertially, the stability is improved. This is achievable by moving the mass centre towards the pivot point. Structural damping, as expected, enhanced the stability. In summary, the parametric study confirms the significance of two parameters which were already neglected in a simpler model. They include damping and the effect of body’s motion in AoA.

\section{References}

Bertin, J.J., Smith, M.L., 1998. Aerodynamics for Engineers, 3rd ed. Prentice-Hall, London, UK.

Bisplinghoff, R., Ashley, H., Halfman, R.L., 1996. Aeroelasticity. Dover Publications, New York, USA.

Blevins, R.D., 2001. Flow-Induced Vibrations. Krieger Publishing Company, Florida, USA.

Braaton, H., Lie, H., Skaugset, K., 2008. Higher Order Modal Response of Riser Fairings, 27th International Conference on Offshore Mechanics and Arctic Engineering (OMAE08-57971). American Society of Mechanical Engineers (ASME), Estoril, Portugal.

Calkins, D.E., 1984. Two-Dimensional Hydrodynamic Characteristics of a Bluff Symmetrical Fairing Section. AIAA Journal 22 (9), 1216-1221.

Ericsson, L.E., Reding, J.P., 1980. Potential Hydroelastic Instability of Profiled Underwater Structures. Journal of Hydronautics 14 (4), 97-104.

Fung, Y.C., 2002. Theory of Aeroelasticity. Dover Publications, New York, USA.

Gardner, T.N., Cole, M.W., 1982. Deepwater Drilling in High Current Environment, Offshore Technology Conference (OTC82-4316), Houston, USA.

Grant, R., Patterson, D., 1977. Riser Fairing for Reduced Drag and Vortex Suppression, Offshore Technology Conference (OTC77-2921), Houston, USA, pp. 343-352.

Grimminger, G., 1945. The Effect of Rigid Guide Vanes on The Vibration and Drag of a Towed Circular Cylinder, Report No 504. David Taylor Model Basin, Washington, USA.

Hoerner, S., 1965. Fluid Dynamic Drag: Practical Information on Aerodynamic Drag and Hydrodynamic Resistance. Hoerner Fluid Dynamics, USA.

Hoerner, S., 1992. Fluid-Dynamic Lift: Practical Information on Aerodynamic and Hydrodynamic Lift. Hoerner Fluid Dynamics, USA. 
Ikeda, Y., Katayama, T., Enomoto, T., 2003. Reduction of Inclination and Vortex-Induced-Oscillation of a Spar Buoy in Rapid Current, 13th International Offshore and Polar Engineering Conference, Honolulu, USA, pp. 248-255.

Khorasanchi, M., 2009. Instability of Riser Fairings in Currents, Department of Naval Architecture and Marine Engineering. Universities of Glasgow and Strathclyde, Glasgow, p. 309.

Lee, L., Allen, D.W., 2005. The Dynamic Stability of Short Fairings, Offshore Technology Conference (OTC05-17125), Houston, USA.

Lee, L., Allen, D.W., Henning, D.L., McMullen, D., 2004. Damping Characteristics of Fairings for Suppressing Vortex-Induced Vibrations, 23rd International Conference on Offshore Mechanics and Arctic Engineering (OMAE04-51209). American Society of Mechanical Engineers (ASME), Vancouver, Canada.

Meyer, P.A., Fort, J.A., Shekarriz, A., Russell, D.A., 1995. Hydrodynamic Performance of an Offshore Drilling Riser Fairing, 3rd International Symposium on Performance Enhancement for Marine Applications, Newport, USA, pp. 141-150.

Slocum, S.T., Ding, Z.J., Frank, W.R., Cox, M.R., 2004. Flutter Instability in Riser Fairings, Offshore Technology Conference (OTC04-16342), Houston, USA.

Spencer, D., Leverette, S., Masters, R.H., Quinn, R., Schaudt, K.J., 2007. Enabling Enhancements of Riser VIV Design Techniques Through Detailed Interpretation of Test Results for VIV Suppression Devices, Offshore Technology Conference (OTC07-18973), Houston, USA. 


\section{Figures}

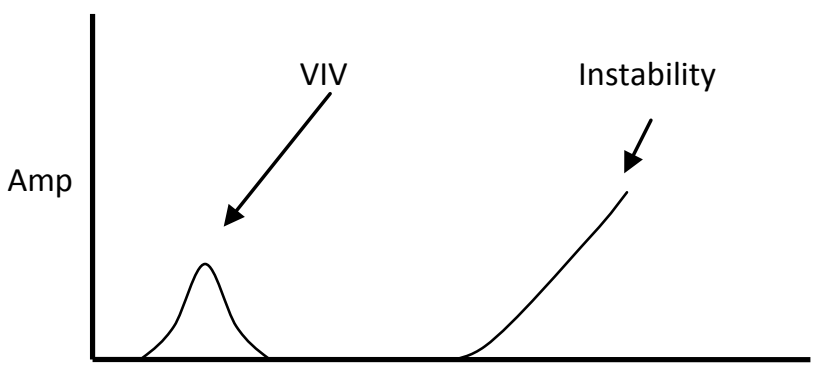

$U_{\mathrm{r}}$

Figure 1 Typical Response of A System vs. Reduced Velocity.
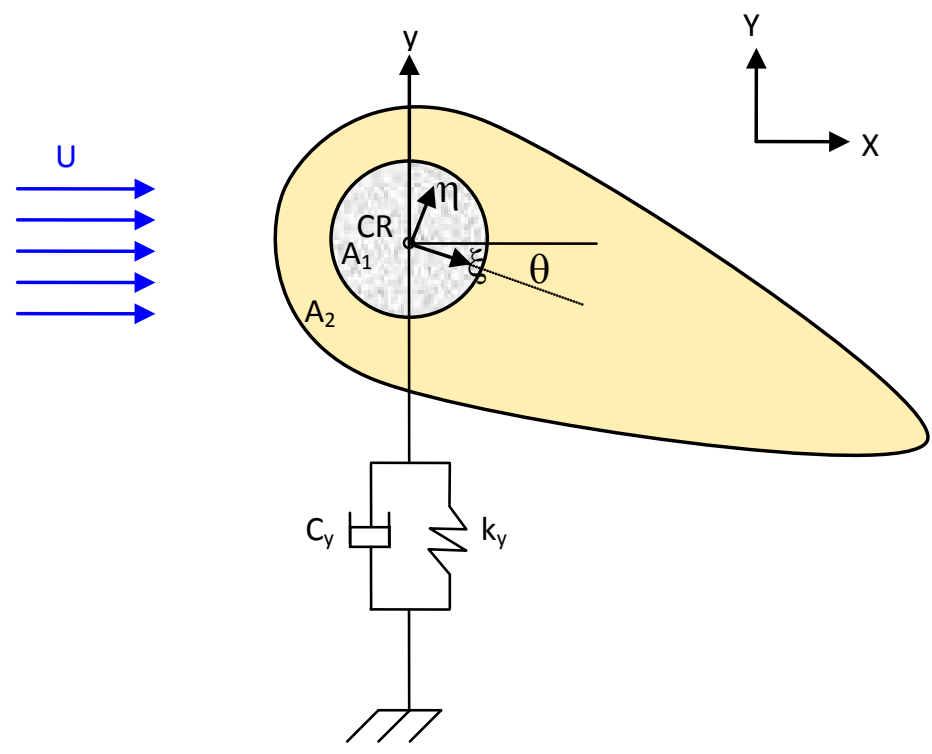

Figure 2 Local and Global Coordinates, Degrees of Freedom. 


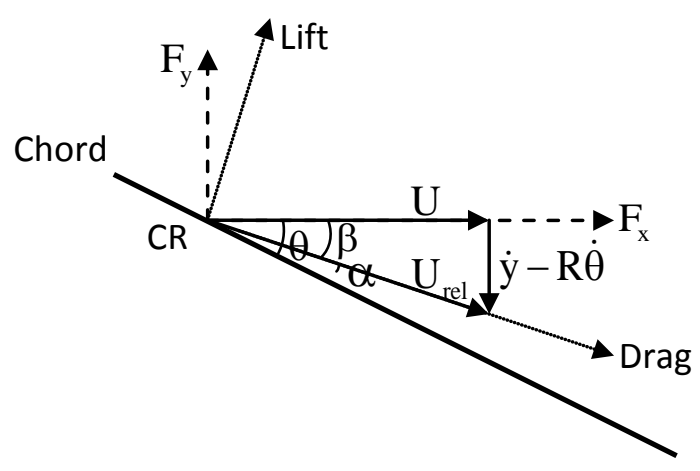

Figure 3 Instantaneous Angle of Attack (AoA).

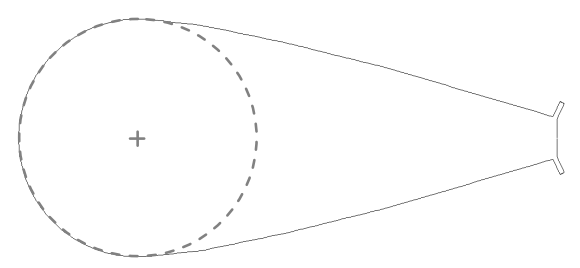

Figure 4 ExxonMobil Fairing on a Rigid Cylinder.

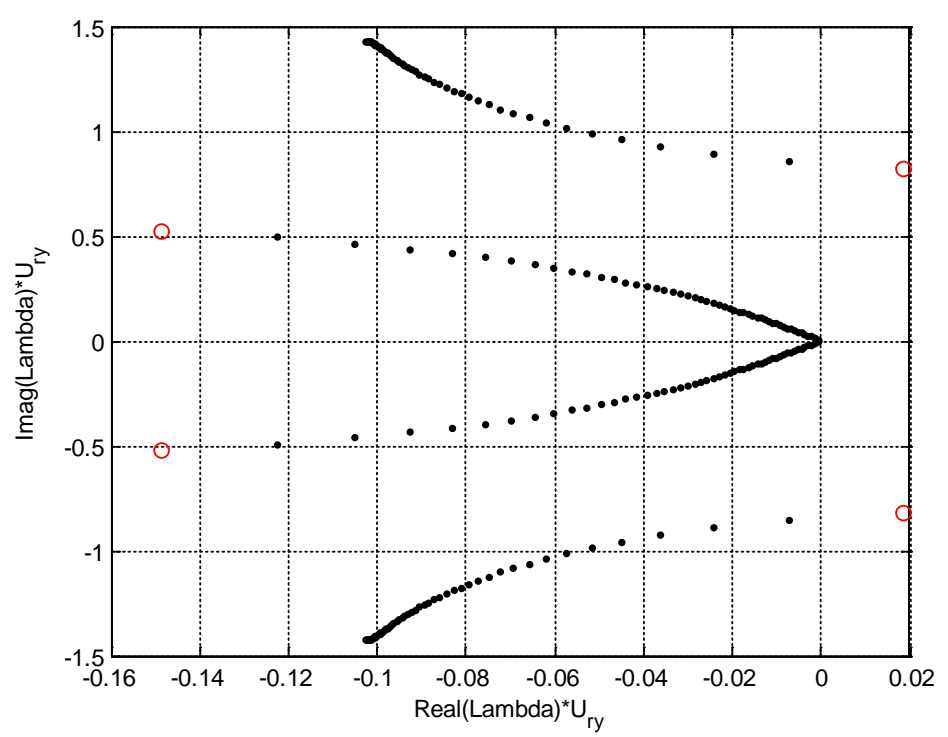

Figure 5 Trend of Eigenvalues by Velocity Increment. 


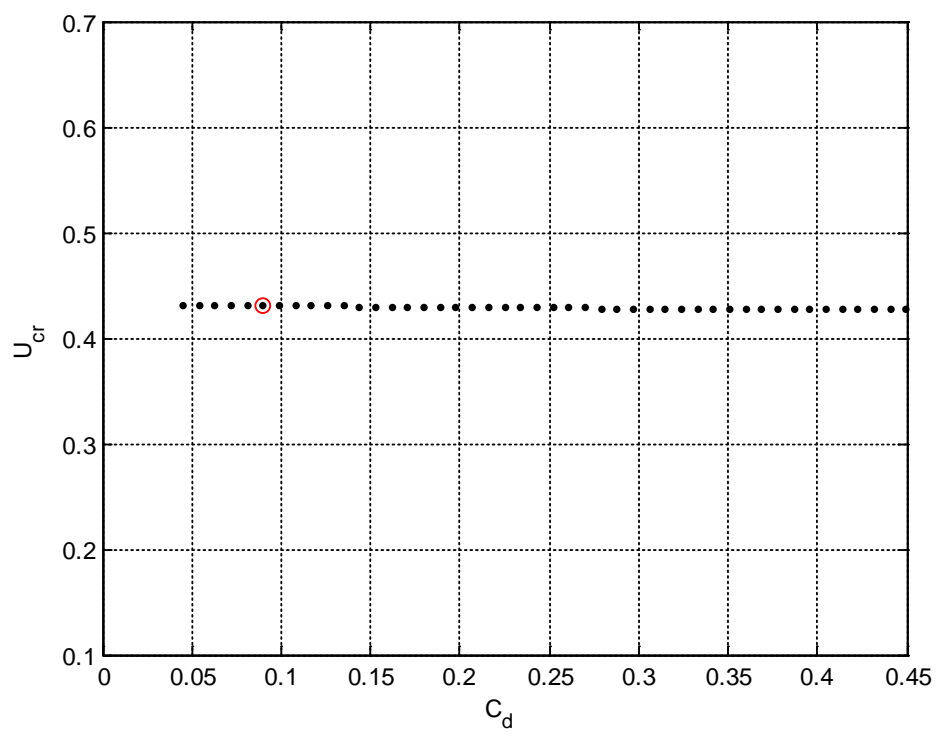

Figure 6 U $_{\text {cr }}$ vs. Drag Coefficient.

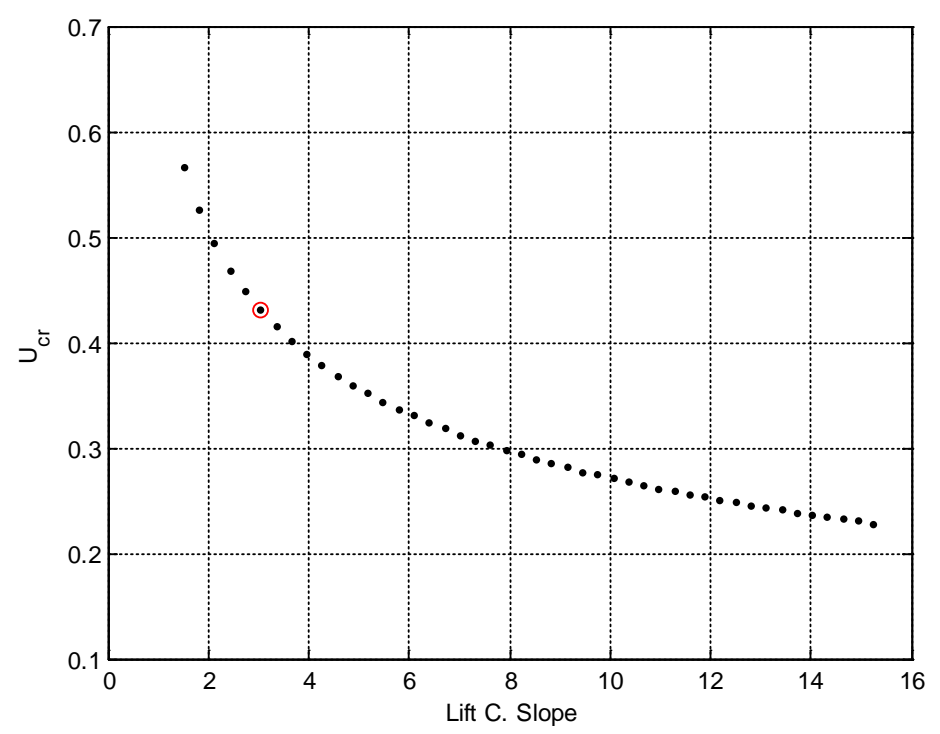

Figure $7 \mathbf{U}_{\mathrm{cr}}$ vs. Lift Coefficient. 


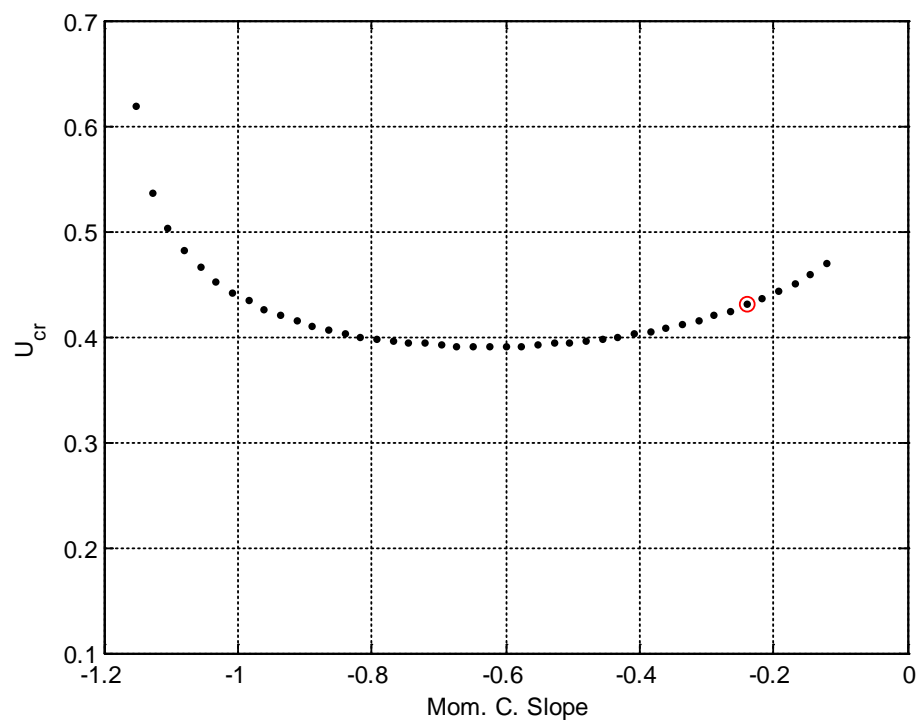

Figure $8 \mathrm{U}_{\mathrm{cr}}$ vs. Moment Coefficient.

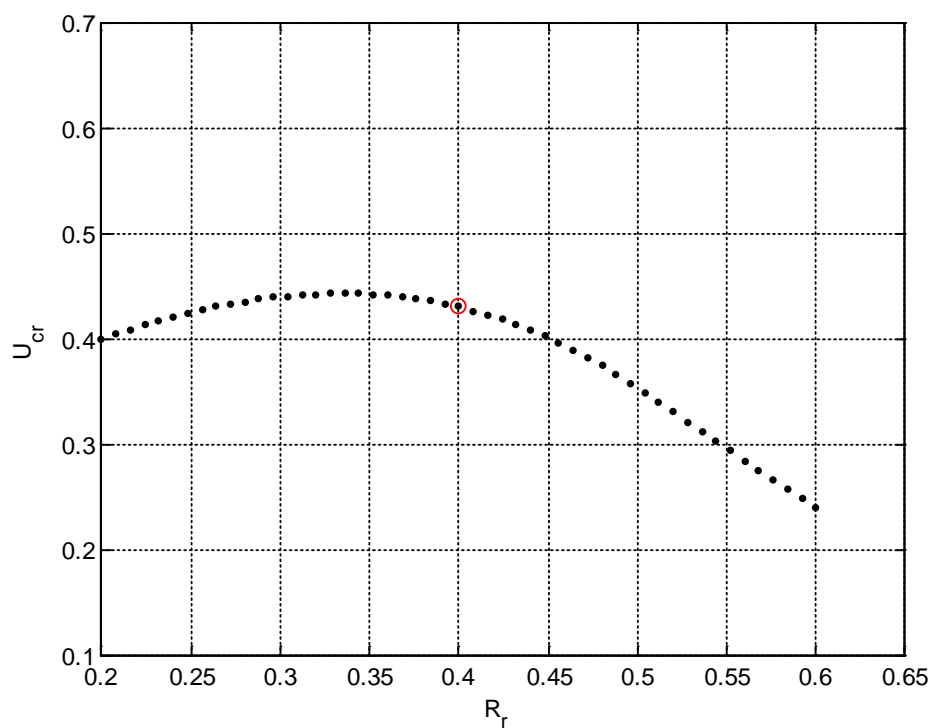

Figure $9 \mathbf{U}_{\mathrm{cr}} \mathbf{v s .} \mathbf{R}_{\mathrm{r}}$. 


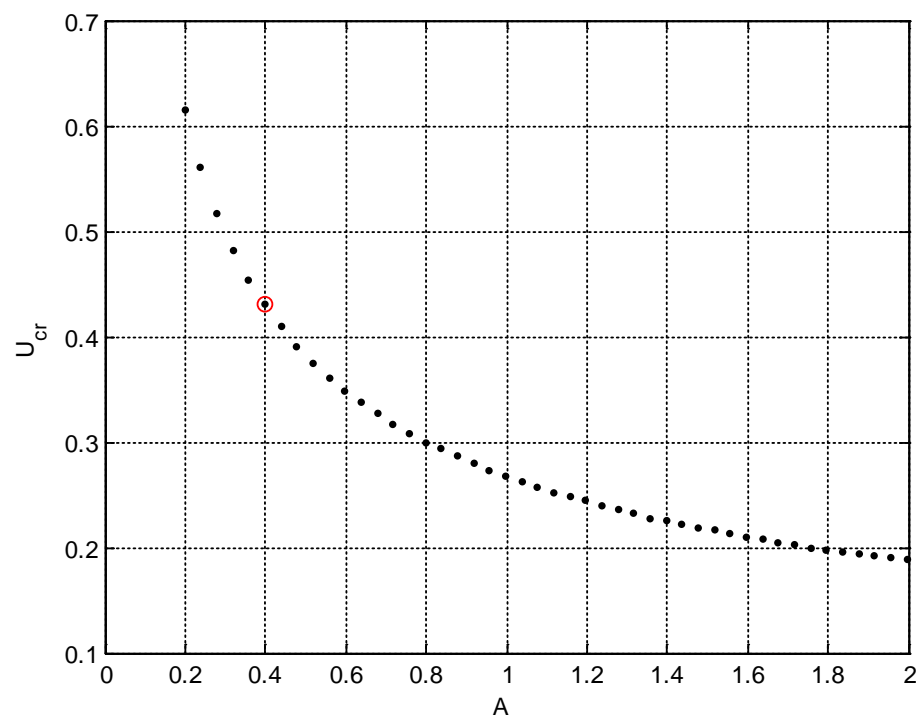

Figure $10 U_{c r}$ vs. $A$.

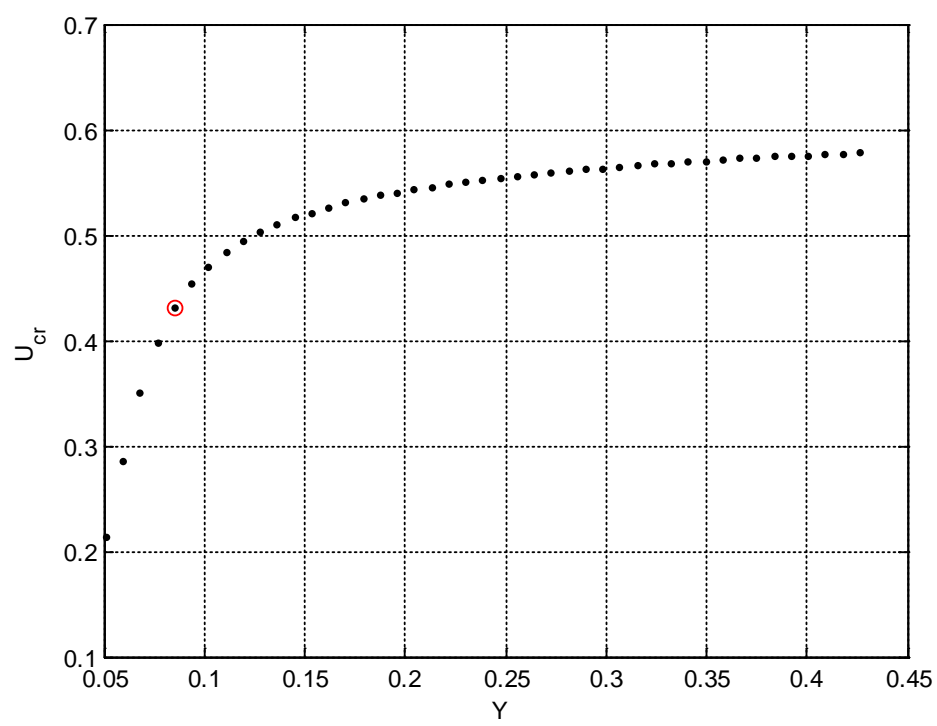

Figure $11 \mathrm{U}_{\text {cr }}$ vs. $\gamma^{2}$. 


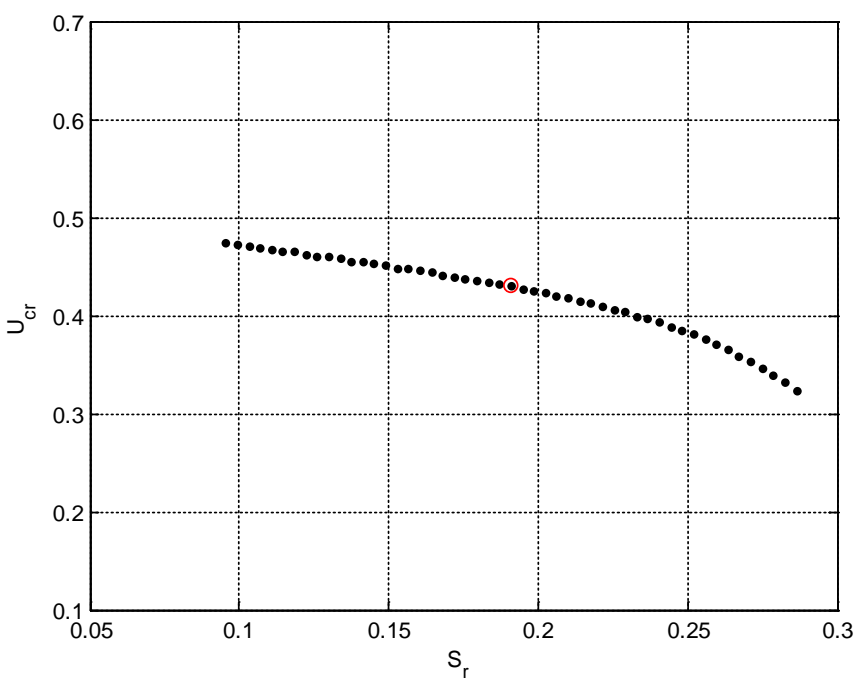

Figure $12 \mathrm{U}_{\mathrm{cr}}$ vs. $\mathrm{S}_{\mathrm{r}}$.

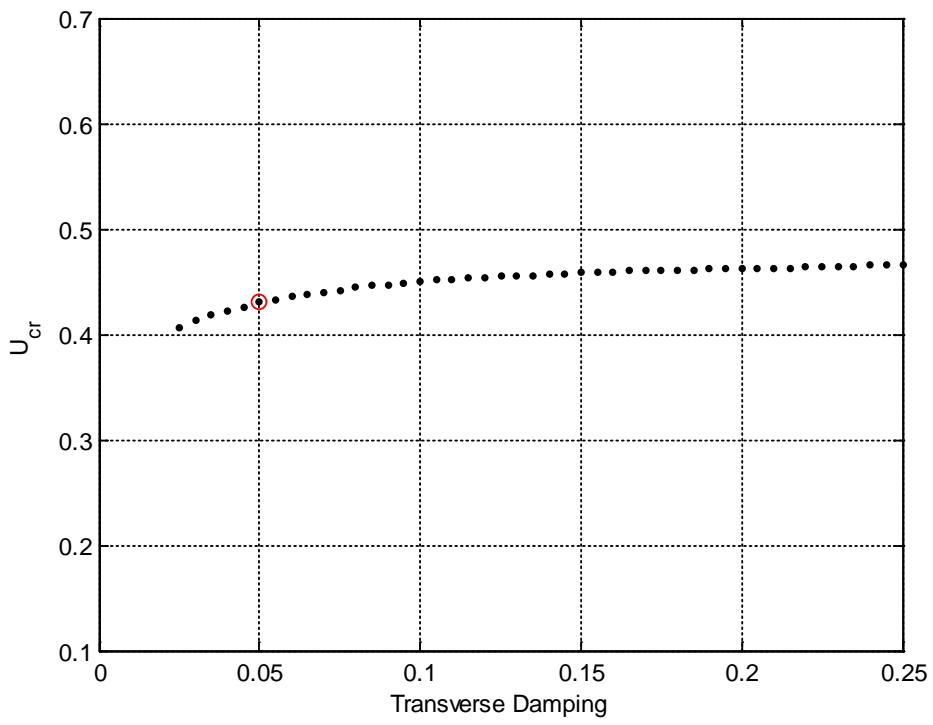

Figure $13 U_{c r}$ vs. Transverse Damping. 


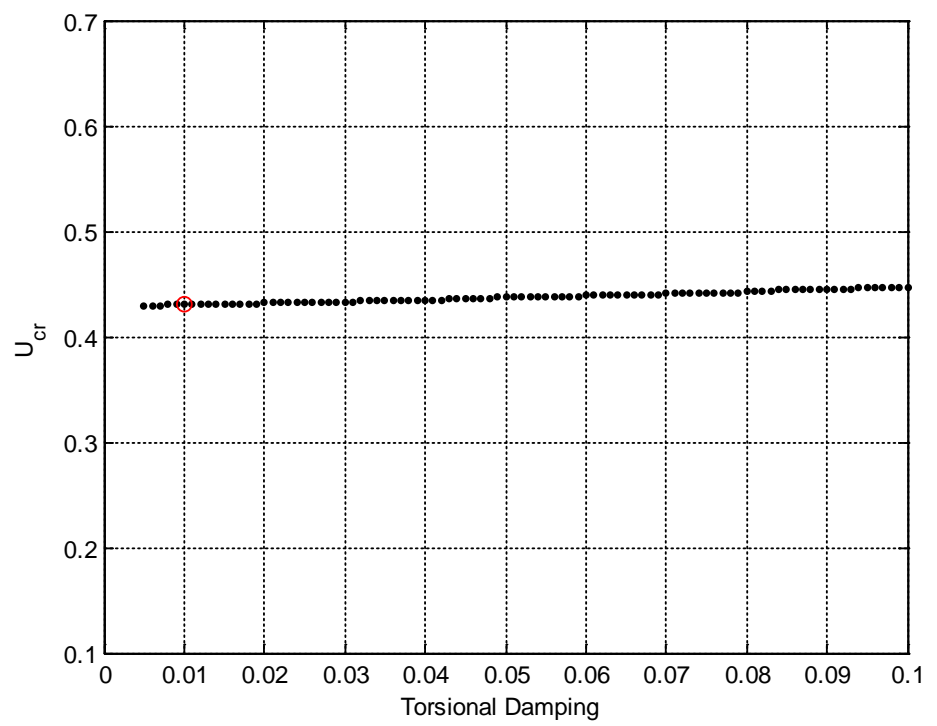

Figure $14 U_{\text {cr }}$ vs. Torsional Damping.

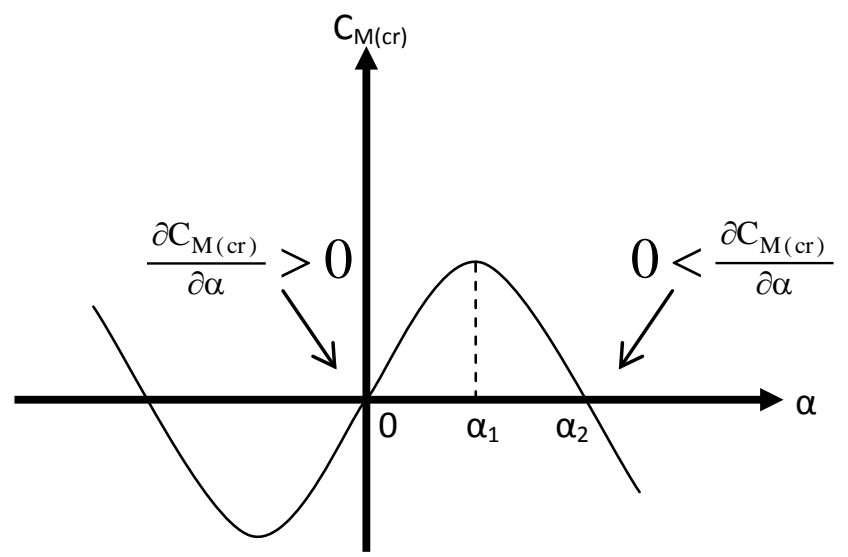

Figure 15 Equilibrium AoA. 